\title{
NONLINEAR MAPPINGS OF NONEXPANSIVE AND ACCRETIVE TYPE IN BANACH SPACES
}

BY FELIX E. BROWDER

Communicated July 21, 1967

Introduction. Let $X$ be a Banach space. If $T$ and $U$ are mappings (in general nonlinear) with domains $D(T)$ and $D(U)$ in $X$ and with values in $X$, then $U$ is said to be nonexpansive if for all $u$ and $v$ in $D(U)$,

$$
\|U(u)-U(v)\| \leqq\|u-v\|,
$$

while $T$ is said to be accretive if for all $u$ and $v$ of $D(T)$,

$$
(T(u)-T(v), w) \geqq 0, \quad w \in J(u-v),
$$

where $(x, w)$ denotes the pairing of the element $x$ of $X$ and the element $w$ of the conjugate space $X^{*}$ and for each $x$ in $X, J(x)$ is the convex subset of $X^{*}$ given by

$$
J(x)=\left\{w \mid w \in X^{*},(w, x)=\|x\|^{2},\|w\|=\|x\|\right\} .
$$

There are two important connections between the classes of nonexpansive and of accretive mappings which give rise to a strong connection between the fixed point theory of nonexpansive mappings and the mapping theory of accretive maps. These are:

(1) If $U$ is a nonexpansive mapping of $D(U)$ into $X$, and if we set $T=I-U, D(T)=D(U)$, then $T$ is an accretive mapping of $D(T)$ into $X$.

(2) If $\{U(t), t \geqq 0\}$ is a semigroup of (nonlinear) mappings of $X$ into $X$ with infinitesimal generator $T$, then all the mappings $U(t)$ are nonexpansive if and only if $(-T)$ is accretive.

For the special case when $X$ is a Hilbert space $H$ (and the concept of an accretive mapping coincides with that of a monotone mapping), the writer in Browder [3], [4] used the observation (1) above and the theory of monotone mappings in Hilbert space to prove the following fixed point theorem for nonexpansive maps: If $C$ is a closed bounded convex subset of the Hilbert space $H, U$ a nonexpansive mapping of $C$ into $C$ which maps the boundary of $C$ into $C$, then $U$ has a fixed point in $C$. This line of argument has also been exploited to yield further results on the existence and calculation of fixed points of nonexpansive mappings in Hilbert space and in the class of Banach spaces having weakly continuous duality mappings (like the spaces $l^{p}$, 
$1<p<\infty$ ), (for which see Browder [5], [7], [8], [9], [13], BrowderPetryshyn [14], [15], and Opial [22]). For more general Banach spaces, it was shown independently in Browder [6], Kirk [20], and Göhde [17] that if $X$ is uniformly convex (or more generally has uniform structure in the sense of Brodski-Milman [2]) and if $U$ is a nonexpansive mapping of a closed bounded convex subset $C$ of $X$ into itself, then $U$ has a fixed point in $C$.

In a preceding note [12], the writer has shown, using the observation (2) that this latter fixed point theorem can be used to obtain very general mapping theorems for nonlinear accretive mappings in $\mathrm{Ba}-$ nach spaces $X$ with both $X$ and $X^{*}$ uniformly convex, results which generalize to this context the principal results of the theory of monotone operators in Hilbert space but by totally different arguments.

It is our object in the present note to apply this new general theory of accretive operators as well as other observations to obtain a substantial strengthening of the fixed point theory of nonexpansive mappings in uniformly convex spaces. Among our results are the following:

THEOREM 1. Let $X$ be a uniformly convex Banach space (or more generally a Banach space with uniform structure), $B$ a closed ball in $X$, $G$ an open subset of $X$ containing $B$. Suppose that $U$ is a nonexpansive mapping of $G$ into $X$ which maps the boundary of $B$ into $B$. Then $U$ has a fixed point in $B$.

We note that for general Banach spaces $X$ the result of Theorem 1 cannot be obtained by composing the mapping $U$ with a retractive contraction map of $X$ onto $B$, since such a retraction mapping may not exist outside of Hilbert spaces for $\operatorname{dim}(X) \geqq 3$ (cf. FigueiredoKarlovitz [16]).

We may extend Theorem 1 to the following more general class of mappings:

Definition 1. If $U$ is a mapping of $D(U)$ in $X$ into $X$, then $U$ is said to be pseudo-contractive if for all $u$ and $v$ of $D(U)$ and all $r>0$,

$$
\|u-v\| \leqq\|(1+r)(u-v)-r(U(u)-U(v))\| .
$$

We note that if $U$ is nonexpansive, then $U$ is pseudo-contractive since

$$
\begin{aligned}
&\|(1+r)(u-v)-r(U(u)-U(v))\| \\
& \geqq(1+r)\|u-v\|-r\|U(u)-U(v)\| .
\end{aligned}
$$

ThEOREM 2. Let $X$ be a uniformly convex Banach space (or more 
generally a Banach space with uniform structure), $B$ a closed ball in $X$, $G$ an open set in $X$ containing $B$. Let $U$ be a pseudo-contractive mapping of $G$ into $X$ such that $U$ maps the boundary of $B$ into $B$. Suppose also that $U$ is demicontinuous and that either (a) $U$ is uniformly continuous in the strong topology on bounded subsets of $X$, or (b) $X^{*}$ is uniformly convex. Then $U$ has a fixed point in $B$.

$\$ 1$ contains the proofs of Theorems 1 and 2 . In $\$ 2$ we consider an extension of Theorem 1 which replaces the closed ball $B$ by a general closed bounded convex subset $C$ of $X$.

Theorem 3. Let $X$ be an uniformly convex Banach space, $C$ a closed bounded convex subset of $X, G$ an open subset of $X$ which contains $C$ and such that $C$ has positive distance from $X-G$. Suppose that $U$ is a nonexpansive mapping of $G$ into $X$ which carries the boundary of $C$ into $C$. Then $U$ has a fixed point in $C$.

In $\$ 3$, we consider the related question of whether one can prove existence theorems for nonlinear accretive operators in Banach spaces $X$ without assuming as in [12] that $X^{*}$ is uniformly convex. We establish below the following theorem by a simple constructive argument.

Theorem 4. Let $X$ be a Banach space, $T$ a Lipschitzian mapping of $X$ into $X$ such that for all $u$ and $v$ of $X$

$$
(T(u)-T(v), w) \geqq c_{0}\|u-v\|^{2}(w \in J(u-v))
$$

with a fixed constant $c_{0}>0$. Then $T$ maps $X$ onto $X$.

Two related results proved in [10] are the following:

THEOREM 5. Let $X$ be a uniformly convex Banach space (or one with uniform structure), $T$ an accretive mapping of $X$ into $X$ which is uniformly continuous in the strong topology on bounded sets of $X$. Suppose that $\|T(u)\| \rightarrow+\infty$ as $\|u\| \rightarrow+\infty$. Then $T$ maps $X$ onto $X$.

Theorem 6. Let $X$ be a uniformly convex Banach space with $X^{*}$ uniformly convex, $T$ a demicontinuous accretive mapping of $X$ into $X$ with $\|T(u)\| \rightarrow+\infty$ as $\|u\| \rightarrow+\infty$. Then $T$ maps $X$ onto $X$.

We remark that Theorem 5 rests upon a refinement of a type of existence theorem for differential equations in Banach spaces first given by Kibenko-Krasnoselski-Mamedov [19] and Mamedov [21].

1. Since every nonexpansive mapping $U$ is both pseudo-contractive and Lipschitzian, Theorem 1 is a special case of Theorem 2. To prove the latter, we apply the following fact ([10], [18]): 
Proposition 1. Let $X$ be a Banach space, $U$ a mapping with domain and range in $X, T=I-U$. Then $U$ is pseudo-contractive if and only if $T$ is accretive.

REMARK. It is shown in [14] that if $X$ is a Hilbert space, $U$ is pseudo-contractive if it satisfies the simpler inequality:

$$
\begin{array}{r}
\|U(u)-U(v)\|^{2} \leqq\|u-v\|^{2}+\|(I-U)(u)-(I-U)(v)\|^{2} \\
(u, v \in D(U)) .
\end{array}
$$

Proof of Theorem 2. By Proposition 1, $T=I-U$ is an accretive mapping of $G$ into $X$. We may assume without loss of generality that $B$ is a closed ball about the origin and that $G$ is star-shaped with respect to the origin. If $S$ is the boundary of $B$, then by hypothesis for each $u$ in $S, U(u)$ lies in $B$. Hence for any $w$ in $J(u)$ for such $u$,

$$
(T(u), w)=(u, w)-(U(u), w) \geqq\|u\|^{2}-\|U(u)\| \cdot\|u\| \geqq 0 .
$$

It is established in [10] that for each $x_{0}$ in $G$, there exists a maximal interval $\left[0, d\left(x_{0}\right)\right)$ with $d\left(x_{0}\right)>0$ and a unique solution on this interval of the differential equation

$$
(d u / d t)(t)=-T(u(t)), \quad 0 \leqq t<d\left(x_{0}\right),
$$

with $u(0)=x_{0}$, the derivative taken in the weak topology of $X$. Moreover as $t \rightarrow d\left(x_{0}\right), u(t)$ either approaches $X-G$ or $\|u(t)\| \rightarrow+\infty$.

If $v$ is any point of $G-B$, there exists $r<1$ such that $u=r v$ lies in $S$. For any $w_{1}$ in $J(v)$, $w=r w_{1}$ lies in $J(u)$. Hence

$\left(T(v), w_{1}\right)=(1-r)^{-1}\left(T(v)-T(u), w_{1}-w\right)+r^{-1}(T(u), w) \geqq 0$,

by the accretiveness of $T$ and the remarks above on $(T(u), w)$. Suppose that $u(t)$ is a solution of the differential equation (1) above lying in $S \cup(G-B)$ in the interval $\left[t_{0}, t_{0}+h\right]$. Then:

$$
\limsup _{k \rightarrow 0^{+}}\left\{\left\|u\left(t_{0}+k\right)\right\|^{2}-\left\|u\left(t_{0}\right)\right\|^{2}\right\} \leqq-(T(u(t)), w) \leqq 0
$$

for any $t$ in this interval and any $w$ in $J(u(t))$. Thus the norm $\|u(t)\|$ does not increase on such an interval. It follows that each solution $u(t)$ of the differential equation (1) beginning at a point of $B$ must continue in $B$. Hence for any $u$ in $B, d(u)=+\infty$.

It follows that we may define $U(t) u$ for any $u$ in $B$ and any $t \geqq 0$ to be equal to $u(t)$ where $u(t)$ is the unique solution of the equation (1) with $u(0)=u$. Since $T$ is accretive, $U(t)$ is a nonexpansive mapping of $B$ into $B$ for any $t \geqq 0$, and $U(t+s)=U(t) U(s)$ for $t, s \geqq 0$. By Theorem 2 of [6], such a commuting family of nonexpansive maps of $B$ must have a common fixed point $u_{0}$ in $B$. For $u_{0}$, we have 


$$
T\left(u_{0}\right)=- \text { weak } \lim _{t \rightarrow 0^{+}}\left\{t^{-1}\left[U(t) u_{0}-u_{0}\right]\right\}=0 . \quad \text { q.e.d. }
$$

2. Proof of Theorem 3. We carry through this proof by a different type of argument.

For each $r>0$, let

$$
C_{r}=\{u \mid u \in X, \operatorname{dist}(u, C)<r\} .
$$

$C_{r}$ is an open convex subset of $X$. By hypothesis, there exists $r>0$ such that $\operatorname{cl}\left(C_{r}\right) \subset G$, where $\operatorname{cl}\left(C_{r}\right)$ denotes the closure of $C_{r}$.

Let $v$ be a point of $C_{r}-C$. Then there exists a point $u$ of $\partial C$, the boundary of $C$, such that $\|v-u\|<r$. Since $U$ is a nonexpansive map, $\|U(v)-U(u)\|<r$. By hypothesis, $U(u)$ lies in $C$ if $u$ lies in $\partial C$. Hence $U(v)$ has distance less than $r$ from $C$, i.e., $U(v)$ lies in $C_{r}$. Thus $U\left(\partial C \cup\left(C_{r}-C\right)\right) \subset C_{r}$.

Since $U$ is nonexpansive and $C$ is bounded, there exists a constant $d_{0}>0$ such that $\|(I-U)(v)\| \leqq d_{0}$ for $v$ in $C$. We choose a constant $s$ with $0<s<1$ such that $s d_{0}<r$, and define a mapping $V$ of $\operatorname{cl}\left(C_{r}\right)$ into $X$ by setting $V(v)=s U(v)+(1-s) v$.

$V$ is a convex linear combination of nonexpansive mappings of $\operatorname{cl}\left(C_{r}\right)$ into $X$ and hence is itself nonexpansive. Since $s>0, V$ has the same fixed points in $\mathrm{cl}\left(C_{r}\right)$ as $U$. For any $v$ in $C_{r}$ which is not in $C$, $V(v)$ is a convex linear combination of points of $C_{\boldsymbol{r}}$ and hence lies in $C_{r}$ itself. If $v$ lies in $C$, then

$$
\|V(v)-v\|=s\|U(v)-v\| \leqq s d_{0}<r,
$$

i.e., $V(v)$ lies in $C_{r}$. Thus $V$ maps $C_{r}$ into $C_{r}$ and by continuity, $V$ maps $\operatorname{cl}\left(C_{r}\right)$ into $\operatorname{cl}\left(C_{r}\right)$. Since $V$ is nonexpansive, it follows from Theorem 1 of [6] that $V$ has a fixed point in $\operatorname{cl}\left(C_{r}\right)$. Since $V$ and $U$ have the same fixed points, $U$ has a fixed point in $\operatorname{cl}\left(C_{r}\right)$.

Let $F$ be the fixed point set of $U$ in $C, F_{r}$ the fixed point set of $U$ in $\operatorname{cl}\left(C_{r}\right)$. Then

$$
F=\bigcap_{r>0} F_{r}
$$

By the above, $F_{r}$ is nonempty for each $r>0$. Moreover, each $F_{r}$ is closed, bounded, and convex and hence weakly compact since $X$ is reflexive. Since the family $\left\{F_{r}\right\}$ has the finite intersection property, $F$ is nonempty, i.e., $U$ has a fixed point in $C$. q.e.d.

3. We shall derive Theorem 4 from the following more general result:

Theorem 7. Let $X$ and $Y$ be two Banach spaces, $T_{0}$ and $T_{1}$ two Lipschitzian mappings of $X$ into $Y$. For each $t$, with $0 \leqq t \leqq 1$, let 


$$
T_{t}=(1-t) T_{0}+t T_{1}
$$

and suppose that for each $t$ in $[0,1]$, there exists a constant $c_{t}$ such that

$$
\|u-v\| \leqq c_{t}\left\|T_{t}(u)-T_{t}(v)\right\|, \quad(u, v \in X) .
$$

Suppose further that $T_{0}$ maps $X$ onto $Y$. Then $T_{1}$ maps $X$ onto $Y$.

Proof of Theorem 7 . Let $H$ be the subset of $[0,1]$ of those values of $t$ for which $T_{t}$ maps $X$ onto $Y . H$ is nonempty since 0 lies in $H$. If, as we show below, there exists a constant $r_{0}>0$ such that for any $t_{0}$ in $H$ the interval of radius $r_{0}$ about $t_{0}$ is also contained in $H$, then $H$ is both open and closed in $[0,1]$ and $H=[0,1]$. It will then follow that $T_{1}$ maps $X$ onto $Y$.

For each $t$ in $[0,1]$, let $c_{t}$ be the minimum constant for which the inequality (2) holds. If $s, t$ lie in $[0,1]$, then

$$
\begin{aligned}
\|u-v\| & \leqq c_{t}\left\|T_{t}(u)-T_{t}(v)\right\| \\
& \leqq c_{t}\left\{\left\|T_{s}(u)-T_{s}(v)\right\|+|t-s|\left\|\left(T_{1}-T_{0}\right)(u)-\left(T_{1}-T_{0}\right)(v)\right\|\right\} \\
& \leqq c_{t}\left\|T_{s}(u)-T_{s}(v)\right\|+c_{t}|t-s| M\|u-v\|
\end{aligned}
$$

where $M$ is the Lipschitz norm of $\left(T_{1}-T_{0}\right)$, which we denote by $\left\|T_{1}-T_{0}\right\|_{\text {Lip. }}$. In particular, if $c_{t}|t-s| M<1$, we have

$$
c_{s} \leqq c_{t}\left(1-c_{t}|t-s| M\right)^{-1}
$$

so that $c_{t}$ is upper semicontinuous on $[0,1]$. Hence there exists a constant $c>0$ such that for all $t$ in $[0,1]$ and all $u$ and $v$ in $X$,

$$
\|u-v\| \leqq c\left\|T_{t}(u)-T_{t}(v)\right\| \text {. }
$$

Suppose $t$ lies in $H$. Then $T_{t}$ maps $X$ onto $Y$ and has a Lipschitzian inverse $R$ mapping $Y$ into $X$. By the inequality (3), $\|R\|_{\mathrm{Lip}_{\mathrm{p}} \leqq c^{-1}}$. Let $r_{0}=c /\left\|T_{1}-T_{0}\right\|_{\text {Lip }}$, and suppose that $|s-t|<r_{0}$. For any $y$ in $Y$, the equation $T_{s}(u)=y$ is equivalent by the substitution $u=R(w)$, for $w$ in $Y$, to the equation in $w$ given by

$$
\begin{aligned}
T_{8} R(w) & =T_{t} R(w)+\left(T_{s}-T_{t}\right) R(w) \\
& =w+S_{s}(w)=y .
\end{aligned}
$$

Here $S_{S}=\left(T_{s}-T_{t}\right) R$ is a Lipschitzian mapping with

$$
\left\|S_{s}\right\|_{\text {Lip }} \leqq\left\|T_{t}-T_{s}\right\|_{\text {Lip }}\|R\|_{\text {Lip }} \leqq\left\|T_{1}-T_{0}\right\|_{\text {Lip }} c^{-1}|t-s|<1 .
$$

Hence $\left(I+S_{\mathrm{s}}\right)^{-1}$ exists and $T_{\mathrm{s}}$ maps $X$ onto $Y$. q.e.d. 
Proof of Theorem 4. We let $T_{0}=I, T_{1}=T$. Both $T_{0}$ and $T_{1}$ are Lipschitzian mappings. If we set $c_{1}=\min \left(c_{0}, 1\right)$, and for any $t$ with $0 \leqq t \leqq 1$, set $T_{t}=(1-t) T_{0}+t T_{1}$, then for any $u$ and $v$ of $X$, we have $\left(T_{t}(u)-T_{t}(v), w\right)=(1-t)(u-v, w)+t(T(u)-T(v), w) \geqq c_{1}\|u-v\|^{2}$ for any $w$ in $J(u-v)$. It follows that

$$
c_{1}\|u-v\|^{2} \leqq\left\|T_{t}(u)-T_{t}(v)\right\| \cdot\|u-v\|
$$

and hence $\|u-v\| \leqq c_{1}^{-1}\left\|T_{t}(u)-T_{t}(v)\right\|$. Since $I$ maps $X$ onto $X$, we may apply Theorem 7 and obtain the fact that $T$ maps $X$ onto $X$.

q.e.d.

\section{BIBLIOGRAPHY}

1. L. P. Belluce and W. A. Kirk, Fixed point theorems for families of contraction mappings, Pacific J. Math. (1966), 213-217.

2. M. S. Brodski and D. P. Milman, On the center of a convex set, Dokl. Akad. Nauk SSSR 59 (1948), 837-840. (Russian)

3. F. E. Browder, Existence of periodic solutions for nonlinear equations of evolution, Proc. Nat. Acad. Sci. U.S.A. 53 (1965), 1100-1103.

4. - Fixed point theorems for noncompact mappings in Hilbert space, Proc. Nat. Acad. Sci. U.S.A. 53 (1965), 1272-1276.

5. - Mapping theorems for noncompact nonlinear operators in Banach spaces, Proc. Nat. Acad. Sci. U.S.A. 54 (1965), 337-342.

6. - Nonexpansive nonlinear operators in a Banach space, Proc. Nat. Acad. Sci. U.S.A. 54 (1965), 1041-1044.

7. - Fixed point theorems for nonlinear semicontractive mappings in Banach spaces, Arch. Rational Mech. Anal. 21 (1966), 259-269.

8. —- Problèmes nonlinéaires, Univ. of Montreal Press, Montreal, 1966.

9. - Convergence of approximants in fixed points of nonexpansive mappings, Arch Rational Mech. Anal. 22 (1967), 82-90.

10. - Nonlinear equations of evolution and the method of steepest descent in Banach spaces (to appear).

11. - Nonlinear accretive operators, Bull. Amer. Math. Soc. 73 (1967), $470-476$.

12. - Nonlinear equations of evolution and nonlinear accretive operators in Banach spaces, Bull. Amer. Math. Soc. 73 (1967), this issue.

13. - Convergence theorems for sequences of nonlinear operators in Banach spaces, Math. Z. 100 (1967), 201-225.

14. F. E. Browder and W. V. Petryshyn, The solution by iteration of nonlinear functional equations in Banach spaces, Bull. Amer. Math. Soc. 72 (1966), 571-575.

15. - Construction of fixed points of nonlinear mappings in Hilbert spaces, J. Math. Anal. Appl. (to appear).

16. D. G. de Figueiredo and L. A. Karlovitz, On the radial projection in normed spaces, Bull. Amer. Math. Soc. 73 (1967), 364-368.

17. D. Göhde, Zum Prinzip der kontraktiven Abbildung, Math. Nachr. 30 (1966), 251-258.

18. T. Kato, Nonlinear semigroups and evolution equations (to appear). 
19. A. V. Kibenke, M. A. Krasnoselski, and Ya. D. Mamedov, One-sided estimates, and conditions for existence of solutions of differential equations in functional spaces, UCen. Zap. Azerbaijan Univ., No. 3 (1961), 13-17.

20. W. A. Kirk, $A$ fixed point theorem of mappings which do not increase distance, Amer. Math. Monthly 72 (1965), 1004-1006.

21. Ya. D. Mamedov, One sided estimates and conditions for existence and uniqueness of solutions of the Cauchy problem in Banach spaces, Sibirsk. Mat. Ž. 6 (1965), 1190-1196.

22. Z. Opial, Weak convergence of the sequences of successive approximants for nonexpansive mappings in Banach spaces, Bull. Amer. Math. Soc. 73 (1967), 591-597.

University of Chicago 